\section{Physiological and Biochemical Responses of Two Herbaceous Peony Cultivars to Drought Stress}

\author{
Qi Wang, Rui Zhao, and Qihang Chen \\ College of Landscape Architecture, Beijing Forestry University, Beijing, \\ 100083, P.R. China
}

Jaime A. Teixeira da Silva

P.O. Box 7, Miki-cho post office, Ikenobe 3011-2, Kagawa-ken, 761-0799, Japan

\section{Liqi Chen}

College of Landscape Architecture, Beijing Forestry University, Beijing, 100083, P.R. China

\section{Xiaonan $\mathbf{Y u}^{1}$ \\ College of Landscape Architecture, Beijing Forestry University, Beijing; Beijing Key Laboratory of Ornamental Plants Germplasm Innovation and Molecular Breeding; National Engineering Research Center for Floriculture; and Beijing Laboratory of Urban and Rural Ecological Environment, Beijing, 100083, P.R. China}

Additional index words. Paeonia lactiflora, drought damage index, leaf relative water contents, osmotic adjustment substance, antioxidant enzymes, hormones

\begin{abstract}
Herbaceous peony is a perennial flowering plant with strong environmental adaptability and may be a good candidate for culture in arid areas. In this study, the physiological and biochemical responses of two herbaceous peony cultivars to different soil moisture gradients in pots were assessed by analyzing changes in 13 stress-related indices. The drought damage index (DDI) and the contents of malondialdehyde (MDA), soluble sugar, proline, and abscisic acid (ABA) generally increased as drought stress intensified, whereas leaf relative water content (LRWC) decreased, and the contents of soluble protein, indole-3-acetic acid (IAA), the ratio of IAA and ABA, and the activities of four antioxidant enzymes fluctuated. For the leaves, a positive correlation was found between DDI and superoxide dismutase (SOD), MDA, soluble sugar, proline, ascorbate peroxidase (APX), and ABA, but it was negatively correlated with LRWC, peroxidase (POD), and catalase (CAT). In fibrous roots, DDI was positively correlated with MDA, soluble sugar, proline, soluble protein, and ABA but was negatively correlated with SOD, CAT, APX, and IAA/ABA. Principal component analysis and subordinate functions were used to evaluate drought resistance of the two cultivars, with 'Karl Rosenfield' showing greater resistance to drought than 'Da Fu Gui'.
\end{abstract}

The shortage of water resources is a serious problem facing the world's agriculture and horticulture. Increasingly severe drought calls for saving water in green spaces and also for more drought-resistant landscape plants. However, the effects of water deficit on plants are manifested in various stages, affecting specific physiological metabolic processes such as absorption and transportation of water and nutrients (Aranda et al., 2015; Smith et al., 2009). These physiological and biochemical processes can reflect a plant's response to environmental stresses

Received for publication 30 Oct. 2018. Accepted for publication $15 \mathrm{Jan} .2019$.

This work was financially supported by the National Natural Science Foundation of China (31400591).

${ }^{1}$ Corresponding author. E-mail: yuxiaonan626@ 126.com.
(Lambers et al., 2008). Paeonia lactiflora, a traditional ornamental plant, has a cultivation history of more than 3900 years and is currently a sought-after cut flower in international markets ( $\mathrm{Li}, 1999)$. With a perennial crown (metamorphosed underground shoot), herbaceous peony has developed special growth patterns to escape stress periods such as considerable changes in temperature, light, and humidity during their annual growth cycle and to resume growth in the usual season, which differentiates it from other perennial plants (Kamenetsky et al., 2003), but the resistance of herbaceous peony varieties cultivated worldwide to these stresses also varies widely.

Only a few studies on the physiological and biochemical responses of herbaceous peonies to drought stress have been published. Guo (2009) studied the phenotypic traits and leaf physiological and biochemical characteristics of four herbaceous peony cultivars treated with different soil moisture content (SMC) $(75 \pm 5 \%, 55 \pm 5 \%, 35 \pm 5 \%$, and $15 \pm 5 \%$ of saturated SMC). Using principal component analysis to comprehensively analyze the indices of the test materials, Guo found that four cultivars could be ranked from strong to weak drought resistance: 'Da Fu Gui', 'Da Hong Pao', 'Zhao Yuan Fen', and 'Mo Zi Lou'. Our team studied the effects of different levels of soil water content on phenotypic traits and water physiological indices of the leaves of some herbaceous peony cultivars such as 'Duchesse de Nemours' that had been introduced to Beijing (Wang et al., 2014a). In many cases, the drought stress response is similar to saline-alkali stress. It indicates that the influence of salt concentration on phenotypic traits is greater than $\mathrm{pH}$ (Wang et al., 2013), although extreme $\mathrm{pH}$ in irrigation water will reduce crown size, leaf area, and stem thickness of herbaceous peonies, which eventually affects the quality of flowers (Zhao et al., 2013).

In contrast, tree peony has received more attention and thus achieved more results in this area of research. Using potted $P$. suffruticosa 'Luo Yang Hong' and 'Wu Long Peng Sheng', Li (2014) assessed the dynamic changes in activity of cell defense enzymes and content of substances related to osmotic adjustment of leaves in naturally induced drought stress and rewatering, finding that after rewatering, most indices of both tree peony cultivars dropped compared with the control. A et al. (2015) used potted plants of three tree peony cultivars with five levels of $\mathrm{SMC}(35 \pm 5 \%, 55 \pm 5 \%, 75 \pm 5 \%, 95 \pm 5 \%$, and half flooded) to study the changes in soluble protein and soluble sugar content, as well as cell membrane permeability under water stress. On the basis of the changes in these indices, a comprehensive assessment of drought resistance of the cultivars was achieved, with cultivars ranked as 'Fen Rong Su Zhuang' > 'Bing Shan Cang Yu' > 'Jin Cheng Nv Lang', whereas their resistance to waterlogging was different. Furthermore, some researchers have conducted detailed studies on the physiology and biochemistry of cultivars of $P$. rockii and $P$. suffruticosa under drought stress (Guo et al., 2014; Kong et al., 2011; Peng et al., 2014).

The objective of this study was to determine how drought stress affects the physiology and biochemistry of two herbaceous peony cultivars. Using relative methods and indices of leaves and fibrous roots, which are more sensitive tissues to environmental changes than leaves, we established a theoretical basis for future screening of drought-resistant varieties for the application of herbaceous peony to arid areas.

\section{Materials and Methods}

Plant material and experimental treatments. The two herbaceous peony cultivars, 'Karl Rosenfield' and 'Da Fu Gui', were collected from nurseries in Xiaotangshan, Beijing. On 28 Sept. 2016, dormant herbaceous peonies 
were pruned to the ground, the roots were lifted out of the ground and washed, and then cut into divisions containing five to eight strong buds and a fleshy root. Roots were shortened to 15 to $20 \mathrm{~cm}$. One hundred plants of each cultivar that had been divided were planted with the same mass of substrate (peat: perlite: vermiculite $=3: 1: 1, \mathrm{v} / \mathrm{v}$ ) in the same size pot. In mid-April of 2017, the buds of all plants were cut off, and all plants were moved into a greenhouse under the following conditions: light intensity: 30 to $1000 \mu \mathrm{mol} \cdot \mathrm{m}^{-2} \cdot \mathrm{s}^{-1}$; photoperiod: $9 \mathrm{~h} \cdot \mathrm{d}^{-1}$; relative humidity: $50 \%$ to $75 \%$; and day/night temperatures: 26 to $30{ }^{\circ} \mathrm{C} / 14$ to $18{ }^{\circ} \mathrm{C}$. On 21 April, a trial experiment was performed with $15 \%$ of the plants, and soil-saturated water content was measured with FieldScout (TDR 100; Spectrum Technologies Inc., Aurora, IL). The division of soil moisture gradients was based on the methods of Guo (2009) and Wang et al. (2014a), and appropriate improvements relied on previous trials. Since phenotypic traits changed rapidly as SMC decreased, a more intensive gradient in the severe drought stress treatment was set: control (CK), mild drought stress (T1), moderate drought stress (T2), moderately severe drought stress (T3), severe drought stress (T4), and very severe drought stress (T5). The soil water content of each treatment was $60 \pm 2 \%, 45 \pm 2 \%, 30 \pm 2 \%, 15$ $\pm 2 \%, 10 \pm 2 \%$, and $5 \pm 2 \%$, respectively, of saturated SMC. The experiment began on 10 May 2017. All plants were irrigated thoroughly. Watering was then stopped to allow soil to dry naturally. SMC was measured with FieldScout at 8:00 to 9:00 AM every morning. Twelve pots were selected randomly from each cultivar that reached the corresponding gradient on the same day, and those already used were no longer used as follow-up experimental materials. In addition to direct determination of phenotypic traits, LRWC, the fibrous roots and leaves of plants were collected randomly and mixed, then wrapped separately in aluminum foil, placed immediately into liquid nitrogen, and stored before the determination of other indices. All the aforementioned experiments were repeated three times.

Measurement methods of indices. Referring to the evaluation system of soil water stress on the physiological and biochemical reactions of herbaceous peony cultivars used by Guo (2009) and Wang et al. (2014a), five levels of scores were assigned based on the degree of drought stress on phenotypic traits (Table 1). The DDI of each cultivar under each treatment was obtained with the following Eq. [1], and the average score of every four plants was calculated as one replicate for further data analysis.

\section{DDI $=\Sigma$ Score of each plant $/$ \\ Number of plants in each treatment}

The LRWC was measured using the method of Barrs and Weatherley (1962). MDA content was determined by the thiobarbituric acid reaction according to Heath and Packer
(1968). Soluble sugar content was measured based on the anthrone method (Morris, 1948) whereas free proline content was quantified using the method of Bates et al. (1973). Soluble protein content was determined using the Coomassie Brilliant Blue G-250 staining method (Bradford, 1976). The measurement and calculation of SOD (EC 1.15.1.1) activity were based on the photochemical method described by $\mathrm{Li}$ (2000). Using the corresponding kit for each assay (Nanjing Jiancheng Bioengineering Institute, Nanjing, China), the activities of POD (EC 1.11.1.7), CAT (EC 1.11.1.6), and APX (EC 1.11.1.11) were measured according to the instructions of each kit. $\mathrm{ABA}$ and IAA were tested with enzyme-linked immunosorbent assay by the College of Agronomy and Biotechnology, China Agricultural University. All indices of each treatment were measured three times.

Statistics. Data of different treatments and indices were subjected to analysis of variance and significant differences $(P \leq 0.05)$ assessed by Duncan's multiple range test (Duncan, 1955) with IBM SPSS Statistics (version 20.0, IBM Corp., Armonk, NY). In this study, correlation analysis among physiological and biochemical indices of leaves and fibrous roots was performed separately. As phenotype intuitively reflects the plant's response to drought stress, the indices whose correlation between it and DDI, which represents the phenotypic trait, is significant at the 0.05 level and 0.01 level were extracted to calculate the eigenvectors and contribution rates $\left(C_{i}\right)$ with principal components analysis. These aforementioned processes also were performed using IBM SPSS Statistics. The membership function method was used to comprehensively evaluate the two herbaceous peony cultivars using a slightly modified method of Wang et al. (2014b) by replacing the original meaning of the reference with drought resistance-related significance. At first, the specific membership function values were calculated with Eq. [2] (if the correlation result between the index and DDI was positive) or with Eq. [3] (if the correlation result between the index and DDI was negative):

$$
\begin{aligned}
& X_{i j}=\left(X-X_{\min }\right) /\left(X_{\max }-X_{\min }\right) \\
& X_{i j}=\left(X_{\max }-X\right) /\left(X_{\max }-X_{\min }\right)
\end{aligned}
$$

where $\mathrm{X}$ is the value of character $\mathrm{j}$ for cultivar $\mathrm{i}, \mathrm{X}_{\min }$ is the minimum value of character $\mathrm{j}$ among all measurements for cultivar $\mathrm{i}, \mathrm{X}_{\max }$ is the maximum value of character $\mathrm{j}$ among all measurements for cultivar $\mathrm{i}$, and $\mathrm{X}_{\mathrm{ij}}$ is the drought-responsive membership value for cultivar $\mathrm{i}$ and character $\mathrm{j}$.

Thereafter, the integrated index $(I)$ of each cultivar was calculated with Eq. [4] (multiplication and addition):

$$
I=\Sigma\left(\mathrm{Ci} \times \mathrm{X}_{\mathrm{ij}}\right)
$$

Finally, the values of ' $I$ ' indicate the level of drought resistance: the lower the value, the greater the drought resistance.

Table 1. Evaluation of phenotypic traits in herbaceous peony under drought stress.

\begin{tabular}{lc}
\hline Description of the external form of the plant & Score \\
\hline No obvious symptoms of drought stress & 1 \\
About $10 \%$ to $30 \%$ of leaves wilt and droop, and their tip and margin turn yellow & 2 \\
About $50 \%$ of the tip and margin of leaves turn yellow, curl and dry & 3 \\
About $70 \%$ to $90 \%$ of leaves curl and dry & 4 \\
Leaves and branches wither until the entire plant dies & 5 \\
\hline
\end{tabular}
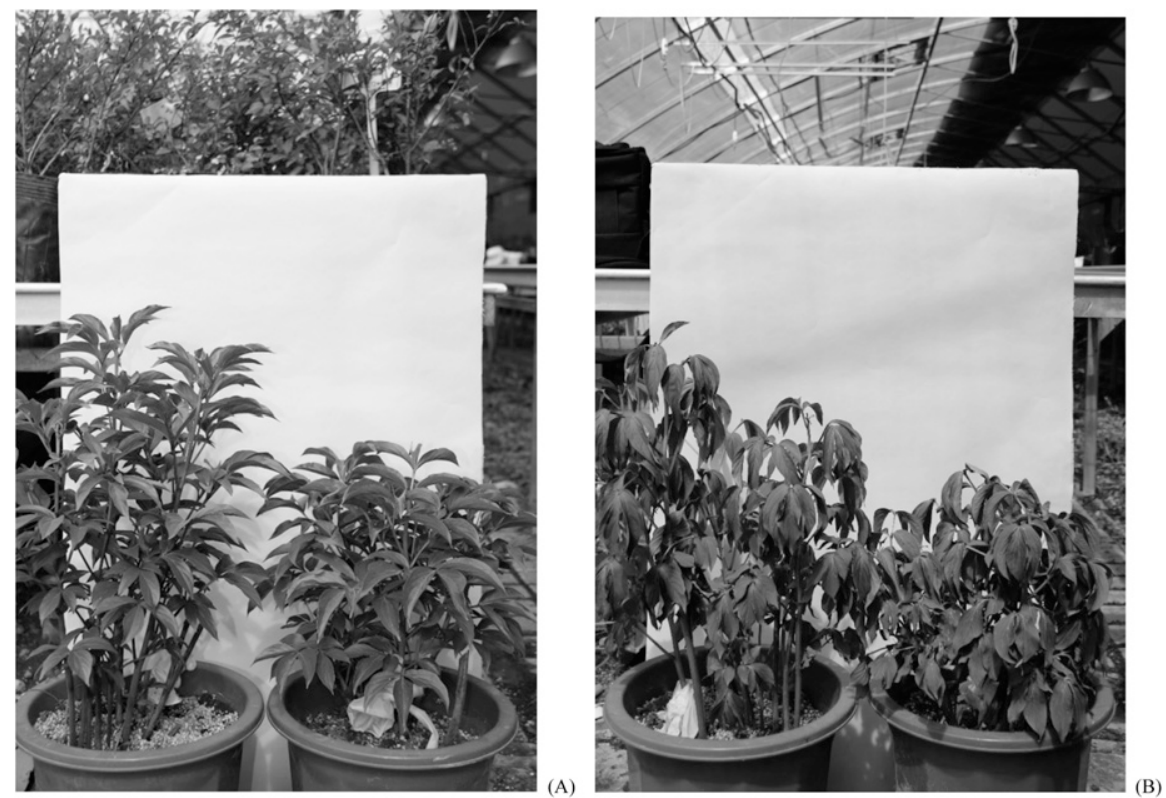

Fig. 1. The appearance of two cultivars. Left is 'Karl Rosenfield' and right is 'Da Fu Gui'. (A) Control (unstressed). (B) T5 (strongly drought stressed). 


\section{Results and Discussion}

Under drought stress, a series of changes take place in plant cells, and the damage is ultimately reflected in the external appearance of plants (Farooq et al., 2009). The degree of drought damage is judged directly by observing the phenotypic traits of plants. To relate phenotypic traits to physiological and biochemical indicators, it is necessary to quantify them. Our experiment showed that as SMC decreased, the phenotypic traits worsened, which was consistent with the results of DDI (Figs. 1 and 2).

LRWC can indicate whether the plant leaf cells are in a state of expansion, which is a commonly used indicator to measure the drought resistance of plants (Raviv and Blom, 2001). As shown in Fig. 3, LRWC of both cultivars declined as SMC decreased. Although there was no significant difference in LRWC between the two cultivars for each treatment, treatments were significantly different for each cultivar, except between T4 and $\mathrm{T} 5$.

MDA content is considered to be an essential indicator of plant resistance to abiotic stress. In Paeonia spp., MDA shows an increase in response to drought stress (A et al., 2015; Guo, 2009; Li, 2014). In our study, MDA accumulated both in leaves and fibrous roots as the severity of drought stress increased (Fig. 4). Water-stress tolerance involves subtle changes in cellular biochemistry and may result from the accumulation of compatible solutes and specific proteins that can be rapidly induced by osmotic stress (Shao et al., 2007). As shown in Fig. 4, the increasing trends of soluble sugar and proline contents observed in both herbaceous peony cultivars under drought stress mirror previous findings of some tuberous root plants such as Dahlia pinnata (Fan et al., 2011), Ipomoea batatas (Yooyongwech et al., 2017), and $P$. suffruticosa 'Luo Yang Hong' and 'Hu Hong' (Kong et al., 2011), the latter using potted plants with four levels of SMC (100\%, $75 \%$ as CK, $50 \%$, and $25 \%$ ). However, the variation in soluble protein content of this study was not unanimous. Guo (2009) found that the soluble protein content of two herbaceous peony cultivars, 'Zhao Yuan Fen' and 'Mo Zi Lou', declined as SMC decreased, whereas 'Da Hong Pao' showed a down-up-down trend. In Medicago sativa, Zhang and Shi (2018) found that soluble protein content first increased then decreased as drought stress increased.

Plants have a protective mechanism against abiotic stresses by synthesizing antioxidants and increasing the activity of antioxidative enzymes (Egert and Tevini, 2002). Guo (2009) studied four herbaceous peony cultivars under drought stress and concluded that their SOD activity first increased then declined with decreasing SMC. In this study, the SOD activity of leaves and fibrous roots of both peony cultivars first increased, then decreased, and finally rose again (Fig. 5). Although the lowest SMC in Guo's experiment was almost the same as T3 in our study, Guo did not test plants growing in soil with lower SMC. Curiously, SOD activity increased slightly from T4 in our study. CAT, which is present only in peroxisomes, transmutes $\mathrm{H}_{2}$ $\mathrm{O}_{2}$ into water and molecular oxygen, whereas POD decomposes $\mathrm{H}_{2} \mathrm{O}_{2}$ through oxidation of cosubstrates such as phenolic compounds and/or antioxidants (Bowler et al., 1994). In this study, the changes in POD and CAT activities in leaves and fibrous roots of the two cultivars fluctuated but were coordinated and synergistic overall, whereas CAT activity decreased continuously throughout severe drought. In general, APX scavenges excess reactive oxygen species and its activity increases under stress, catalyzing the conversion of $\mathrm{H}_{2} \mathrm{O}_{2}$ to water using ascorbate as substrate (Hossain et al., 2012). In a study on the evaluation of heat resistance in
P. lactiflora by Zhang et al. (2016), APX was considered a reliable physiological evaluation index of heat damage in leaves, so we adopted it. In our experiment, the changes in APX activity in leaves was more regular than in fibrous roots, which may be because APX mainly exists in the chloroplast and cytoplasm and is a key enzyme for scavenging $\mathrm{H}_{2} \mathrm{O}_{2}$ in chloroplasts (Caverzan et al., 2016). On the basis of the changes observed in the activity of these four enzymes, we believe that although the antioxidant enzyme system might play a role in assisting the plant in response to severe drought stress, it was damaged at early growth stages.

Plants have developed many adaptive strategies to enhance drought stress tolerance, including changes in phytohormones (Davies, 2010). ABA regulates stomatal

\section{DDI}

$\square$ 'Karl Rosenfield’ $\square$ 'Da Fu Gui'

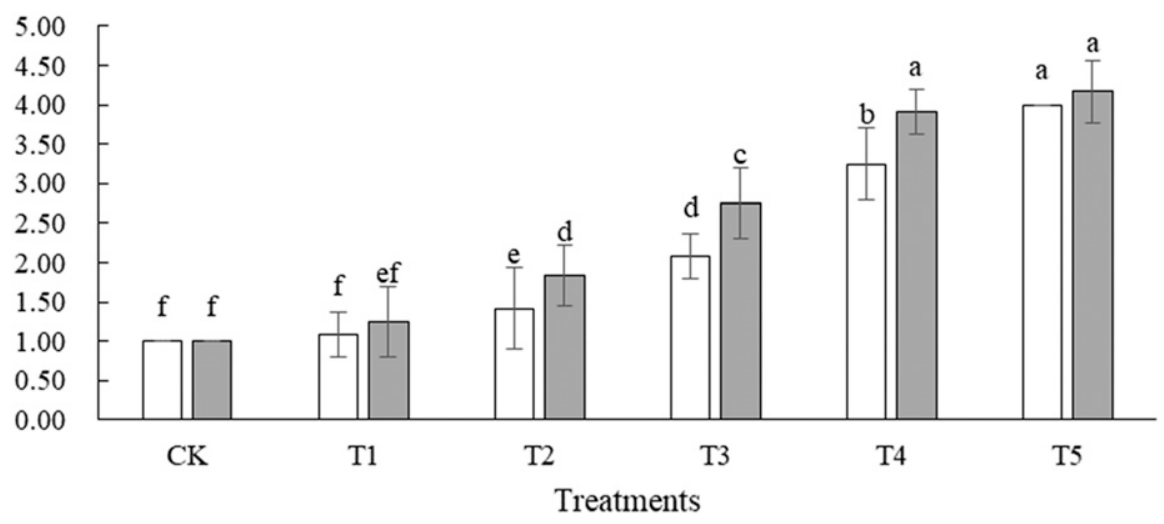

Fig. 2. Drought damage index (DDI) of two herbaceous peony cultivars under drought stress. Values are means of three replicates and error bars represent SD. Different letters on the columns indicate significant differences among treatments across both cultivars according to Duncan's multiple range test at $P \leq 0.05$. $\mathrm{CK}=$ control; $\mathrm{T} 1=$ mild drought stress; $\mathrm{T} 2=$ moderate drought stress; $\mathrm{T} 3=$ moderately severe drought stress; T4 = severe drought stress; $\mathrm{T} 5=$ very severe drought stress.

\section{LRWC (\%)}

\section{$\square$ 'Karl Rosenfield’ $\square$ 'Da Fu Gui'}

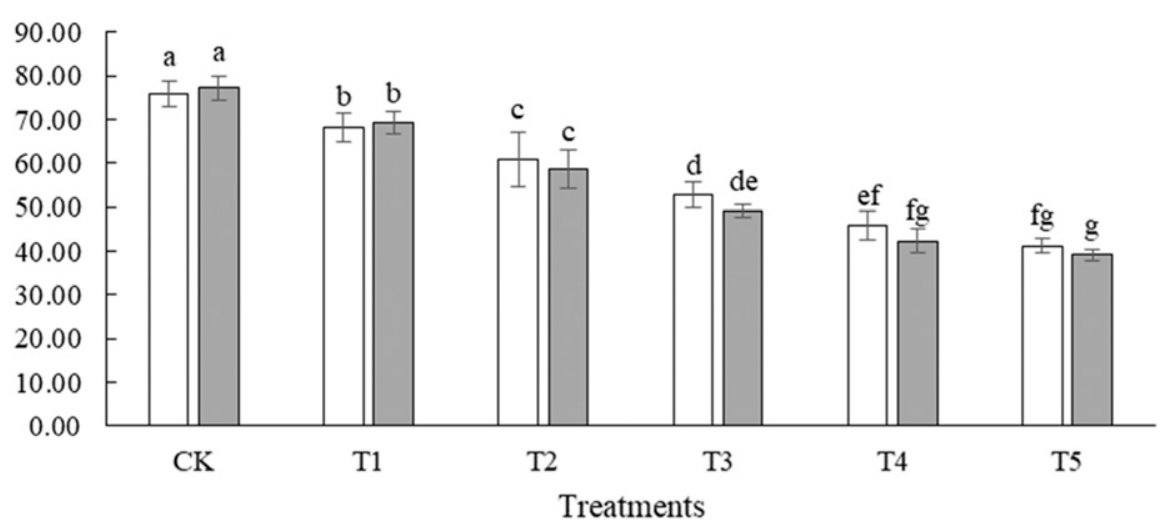

Fig. 3. Leaf relative water contents (LRWC) of two herbaceous peony cultivars under drought stress Values and different letters on the columns indicate the same meanings as Fig. 2. $\mathrm{CK}=$ control; $\mathrm{T} 1=$ mild drought stress; $\mathrm{T} 2=$ moderate drought stress; $\mathrm{T} 3=$ moderately severe drought stress; $\mathrm{T} 4=$ severe drought stress; T5 = very severe drought stress. 

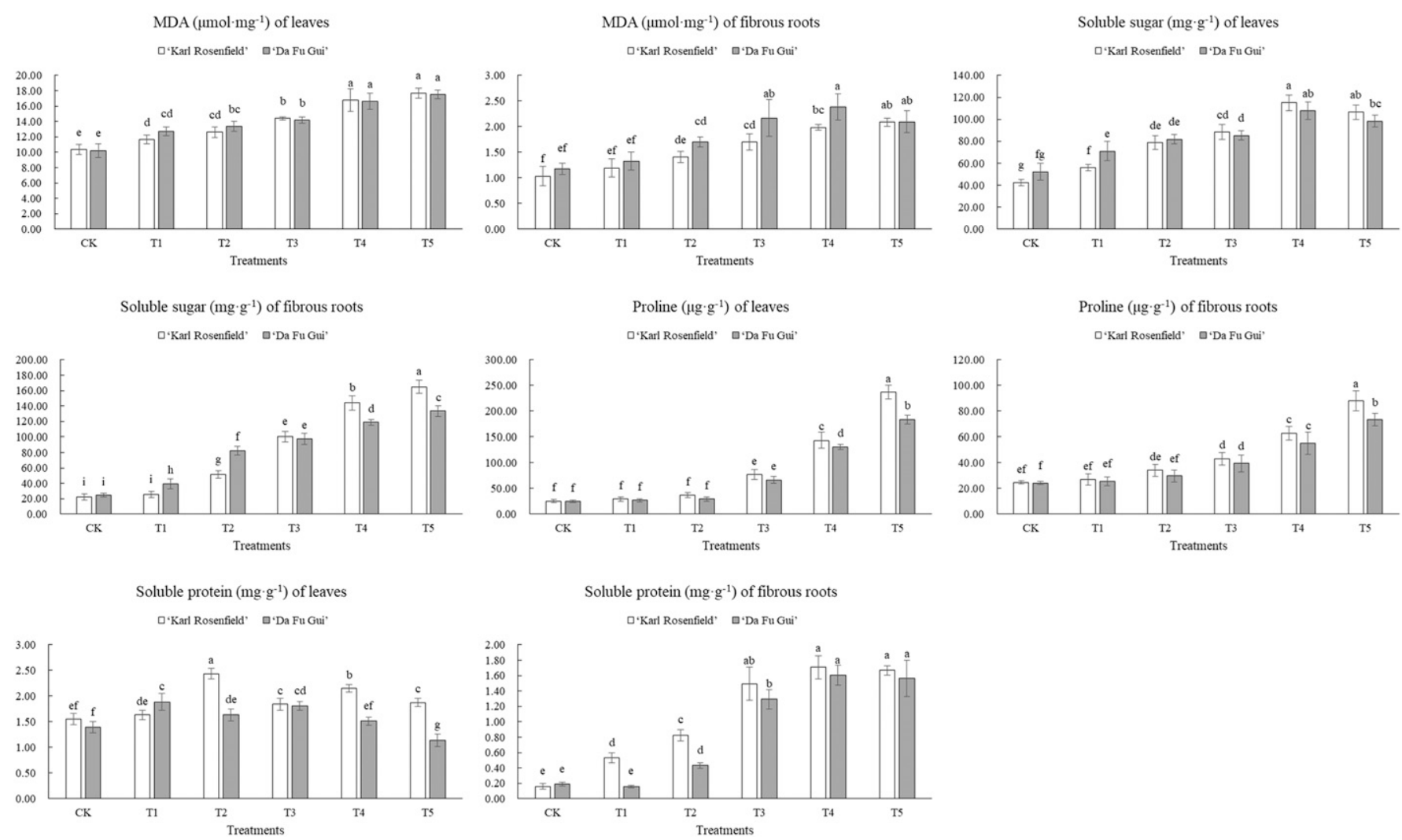

Fig. 4. Contents of malondialdehyde (MDA), soluble sugar, proline, and soluble protein of two herbaceous peony cultivars under drought stress. Values are means of three biological replicates and error bars represent SD. Different letters on the columns indicate significant differences among treatments across both cultivars of each index according to Duncan's multiple range test at $P \leq 0.05 . \mathrm{CK}=$ control; $\mathrm{T} 1=$ mild drought stress; T2 $=$ moderate drought stress; T3 $=$ moderately severe drought stress; T4 = severe drought stress; T5 = very severe drought stress.
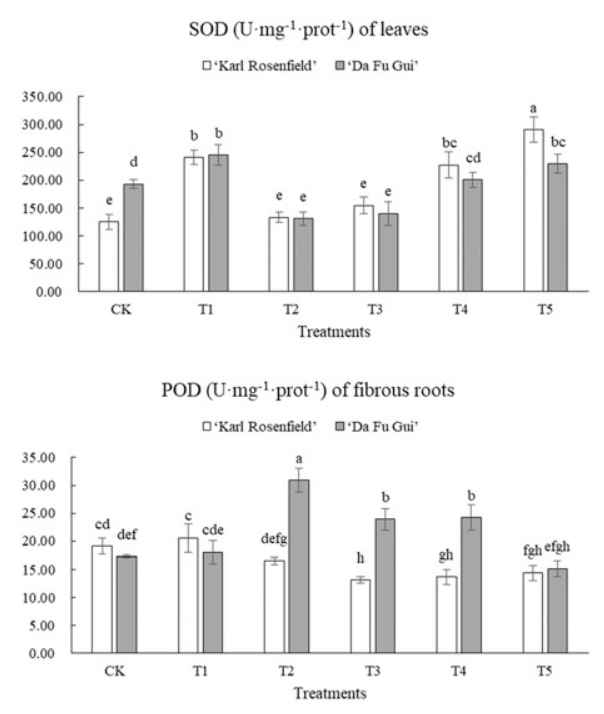

$\operatorname{APX}\left(\mathrm{U} \cdot \mathrm{mg}^{-1} \cdot \operatorname{prot}^{-1}\right)$ of leaves

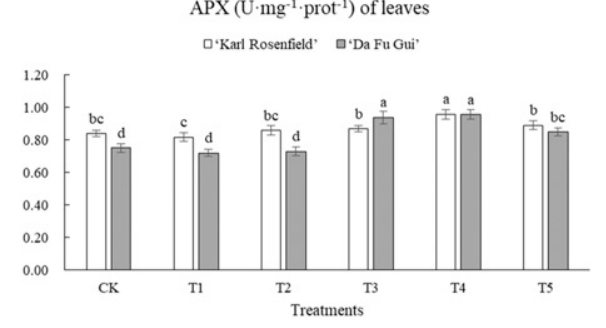

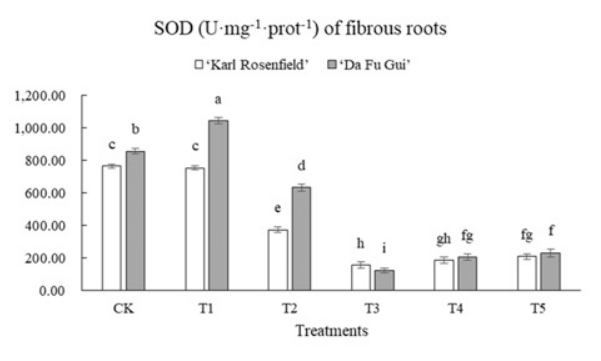
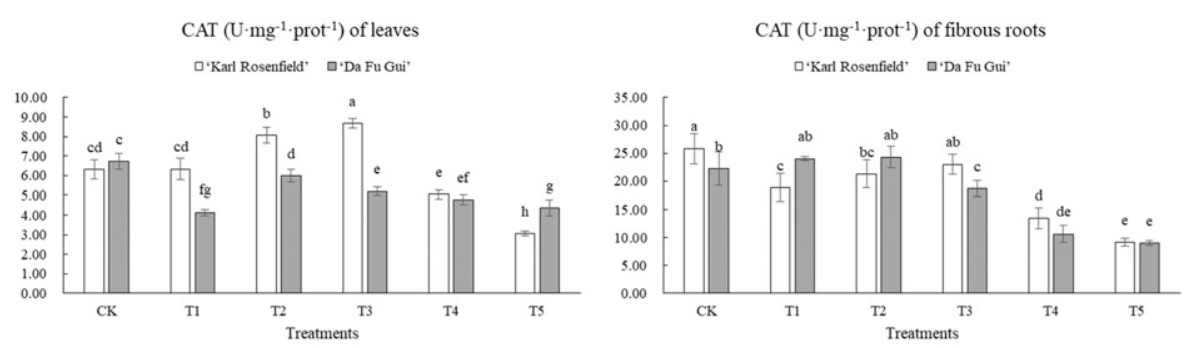

POD $\left(\mathrm{U} \cdot \mathrm{mg}^{-1} \cdot \mathrm{prot}^{-1}\right)$ of leaves

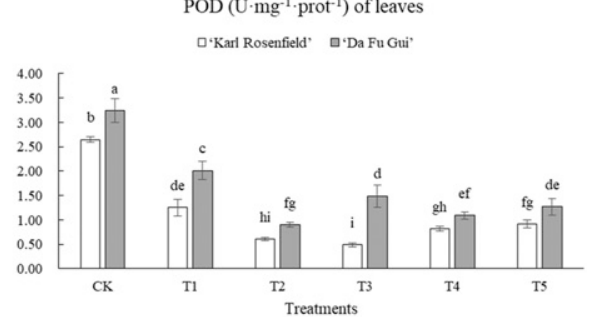

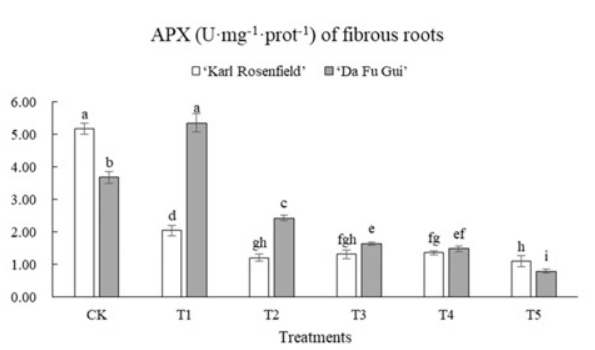

Fig. 5. Activities of superoxide dismutase (SOD), peroxidase (POD), catalase (CAT), and ascorbate peroxidase (APX) of two herbaceous peony cultivars under drought stress. Values and different letters on the columns indicate the same meanings as Fig. 4 . $\mathrm{CK}=$ control; $\mathrm{T} 1=$ mild drought stress; $\mathrm{T} 2=$ moderate drought stress; T3 = moderately severe drought stress; T4 = severe drought stress; T5 = very severe drought stress. 

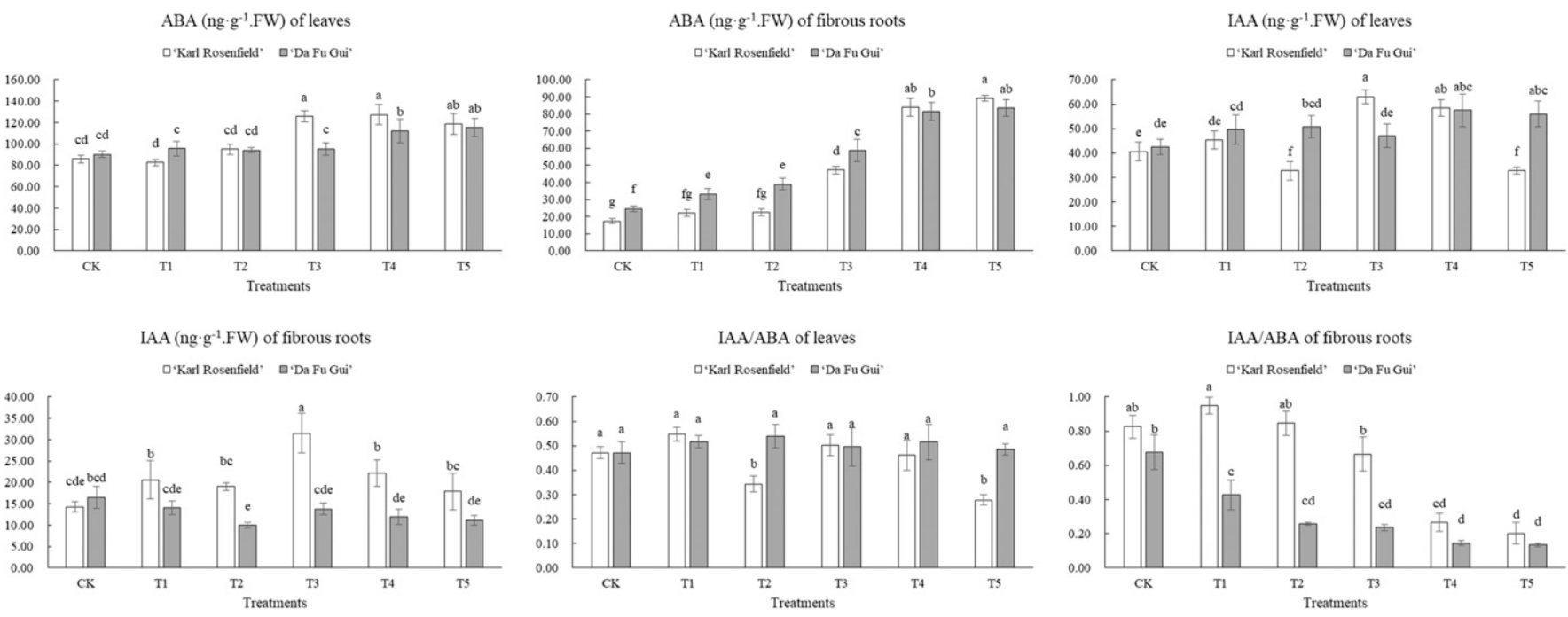

Fig. 6. Contents of abscisic acid (ABA), indole-3-acetic acid (IAA), and IAA/ABA of two herbaceous peony cultivars under drought stress. Values and different letters on the columns indicate the same meanings as Fig. $4 . \mathrm{CK}=$ control; $\mathrm{T} 1=$ mild drought stress; T2 = moderate drought stress; $\mathrm{T} 3=$ moderately severe drought stress; $\mathrm{T} 4=$ severe drought stress; $55=$ very severe drought stress.

Table 2. Correlation analysis between DDI and other drought-responsive physiological and biochemical indices of leaves and fibrous roots (pooled for both herbaceous peony cultivars).

\begin{tabular}{llccccccccccccccc}
\hline & Indices & LRWC & MDA & Soluble sugar & Proline & Soluble protein & SOD & POD & CAT & APX & ABA & IAA & IAA/ABA \\
\hline DDI & Leaves & $-0.934^{* *}$ & $0.930^{* *}$ & $0.859^{* *}$ & $0.919^{* *}$ & -0.152 & $0.410^{*}$ & $-0.465^{* *}$ & $-0.590^{* *}$ & $0.625^{* *}$ & $0.697^{* *}$ & 0.286 & -0.239 \\
& Fibrous roots & - & $0.885^{* *}$ & $0.934^{* *}$ & $0.904^{* *}$ & $0.874^{* *}$ & $-0.778^{* *}$ & -0.134 & $-0.881^{* *}$ & $-0.675^{* *}$ & $0.970^{* *}$ & -0.141 & $-0.790^{* *}$ \\
\hline
\end{tabular}

** Correlation is significant at the 0.01 level (2-tailed).

* Correlation is significant at the 0.05 level (2-tailed).

$\mathrm{DDI}=$ drought damage index $; \mathrm{LRWC}=$ relative water content $; \mathrm{MDA}=$ malondialdehyde $; \mathrm{SOD}=$ superoxide dismutase $; \mathrm{POD}=$ peroxidase $; \mathrm{CAT}=$ catalase;

$\mathrm{APX}=$ ascorbate peroxidase; $\mathrm{ABA}=$ abscisic acid; IAA = indole-3-acetic acid.

Table 3. Eigenvectors and contribution rates of principal components of drought-responsive indices of leaves and fibrous roots.

\begin{tabular}{lccc}
\hline & \multicolumn{2}{c}{ Leaves } & Fibrous roots \\
\cline { 2 - 3 } Indices & Component 1 & Component 2 & Component \\
\hline LRWC & -0.945 & 0.147 & - \\
MDA & 0.973 & 0.026 & 0.890 \\
Soluble sugar & 0.939 & -0.128 & 0.965 \\
Proline & 0.914 & 0.232 & 0.904 \\
Soluble protein & - & - & 0.946 \\
SOD & 0.487 & 0.726 & -0.871 \\
POD & -0.643 & 0.535 & - \\
CAT & -0.515 & -0.756 & -0.861 \\
APX & 0.660 & -0.342 & -0.795 \\
ABA & 0.828 & -0.181 & 0.957 \\
IAA & - & - & - \\
IAA/ABA & - & - & -0.742 \\
Contribution rate/\% & 62.165 & 18.070 & 78.182 \\
Cumulative contribution rate/\% & 62.165 & 80.235 & 78.182 \\
\hline
\end{tabular}

LRWC $=$ relative water content MDA $=$ malondialdehyde; $\mathrm{SOD}=$ superoxide dismutase; $\mathrm{POD}=$ peroxidase; $\mathrm{CAT}=$ catalase; $\mathrm{APX}=$ ascorbate peroxidase; $\mathrm{ABA}=$ abscisic acid; IAA $=$ indole- 3 -acetic acid.

conductance by reducing transpiration water loss and eventually affects the regulation of water balance and osmotic stress tolerance in plants (Morkunas et al., 2014). In our experiment, an increasing trend in $\mathrm{ABA}$ content with more acute drought stress (Fig. 6) is consistent with previous studies in Agrostis spp. (Dacosta and Huang, 2007) and Vaccinium darrowii seedlings (Chen et al., 2017). Under drought stress, IAA also is involved in the physiological responses of plants (Mahouachi et al., 2007). The change in IAA content in our research was irregular, but contradictory results were found regarding changes in IAA content in different plants under different drought stress conditions. With increasing drought stress, IAA content first increased then declined in Agrostis stolonifera ( $\mathrm{Li}$ et al., 2015) and decreased in leaves of Manihot esculenta, but the content in roots varied, depending on the variety (Zhou et al., 2013). In this experiment, IAA/ABA fluctuated in leaves and decreased in fibrous roots, which had the same association in the roots of cassava varieties (Zhou et al., 2013). In contrast, Li et al. (2010) found a low IAA/ $\mathrm{ABA}$ ratio in the main stem leaves of cotton (Gossypium spp.) at an early stage of drought stress, which increased in intermediate and later stages.

The correlation analysis is shown in Table 2 . For the leaves, DDI was positively correlated with SOD $(P \leq 0.05)$, as well as MDA, soluble sugar, proline, APX, and ABA $(P \leq 0.01)$, and was negatively correlated with LRWC, POD, and CAT $(P \leq 0.01)$. In the fibrous roots, DDI was positively correlated with MDA, soluble sugar, proline, soluble protein, and $\mathrm{ABA}(P \leq 0.01)$ and was negatively correlated with SOD, CAT, APX, and IAA/ABA $(P \leq 0.01)$. The former nine indices and the latter nine indices were extracted to calculate the eigenvectors and levels of contribution of principal components of drought-responsive indices of leaves and fibrous roots (Table 3 ). On the basis of membership function values (Table 4), integrated indices were calculated with Eq. [4], and the drought resistance of 'Karl Rosenfield' was ranked as being greater than ' $\mathrm{Da} \mathrm{Fu}$ Gui' (Table 5).

In this study, drought stress triggered a series of reactions in two herbaceous peony cultivars. We have measured the physiological and biochemical indicators of both leaves and fibrous roots of herbaceous peony under drought stress, which would provide a theoretical basis for related mechanisms and 
Table 4. Membership function values of drought-responsive indices of leaves and fibrous roots.

\begin{tabular}{lcccr}
\hline & \multicolumn{2}{c}{ Leaves } & \multicolumn{2}{c}{ Fibrous roots } \\
\cline { 2 - 5 } Indices & 'KR' & 'DFG' & 'KR' & 'DFG' \\
\hline LRWC & 0.000 & 1.000 & - & 1.000 \\
MDA & 0.000 & 1.000 & 0.000 & 0.000 \\
Soluble sugar & 0.000 & 1.000 & 1.000 & 0.000 \\
Proline & 0.000 & 1.000 & 1.000 & 0.000 \\
Soluble protein & - & - & 1.000 & 0.000 \\
SOD & 0.000 & 1.000 & 1.000 & - \\
POD & 0.000 & 1.000 & - & 1.000 \\
CAT & 0.000 & 1.000 & 0.000 & 0.000 \\
APX & 1.000 & 0.000 & 1.000 & 1.000 \\
ABA & 1.000 & 0.000 & 0.000 & - \\
IAA & - & - & - & 1.000 \\
IAA/ABA & - & - & 0.000 & \\
'KR' = Karl Rosenfield; 'DFG' $=$ Da Fu Gui; LRWC $=$ relative water content; MDA = malondialdehyde; \\
SOD = superoxide dismutase; POD = peroxidase; CAT = catalase; APX = ascorbate peroxidase; ABA $=$ \\
abscisic acid; IAA = indole-3-acetic acid.
\end{tabular}

Table 5. Integrated index and relative drought resistance precedence of two herbaceous peony cultivars.

\begin{tabular}{|c|c|c|c|c|}
\hline \multirow[b]{2}{*}{ Indices } & \multicolumn{2}{|c|}{ 'KR' } & \multicolumn{2}{|c|}{ 'DFG' } \\
\hline & Leaves & Fibrous roots & Leaves & Fibrous roots \\
\hline$\overline{\mathrm{LRWC}}$ & 0.000 & - & 0.127 & - \\
\hline MDA & 0.000 & 0.000 & 0.126 & 0.112 \\
\hline Soluble sugar & 0.000 & 0.122 & 0.125 & 0.000 \\
\hline Proline & 0.000 & 0.114 & 0.126 & 0.000 \\
\hline Soluble protein & - & 0.119 & - & 0.000 \\
\hline SOD & 0.000 & 0.110 & 0.090 & 0.000 \\
\hline POD & 0.000 & - & 0.102 & - \\
\hline CAT & 0.000 & 0.000 & 0.094 & 0.109 \\
\hline APX & 0.097 & 0.100 & 0.000 & 0.000 \\
\hline $\mathrm{ABA}$ & 0.113 & 0.000 & 0.000 & 0.121 \\
\hline IAA & - & - & - & - \\
\hline IAA/ABA & - & 0.000 & - & 0.094 \\
\hline Integrated index & \multicolumn{2}{|c|}{0.775} & \multicolumn{2}{|c|}{1.226} \\
\hline Precedence & \multicolumn{2}{|c|}{1} & \multicolumn{2}{|c|}{2} \\
\hline
\end{tabular}

'KR' = Karl Rosenfield; 'DFG' = Da Fu Gui; LRWC = relative water content; MDA = malondialdehyde; $\mathrm{SOD}=$ superoxide dismutase $\mathrm{POD}=$ peroxidase CAT $=$ catalase $; \mathrm{APX}=$ ascorbate peroxidase $; \mathrm{ABA}=$ abscisic acid; IAA = indole-3-acetic acid.

allow for the improvement of herbaceous peony introduction to drought stressed zones. A comprehensive evaluation of drought resistance between the two cultivars is necessary because the trends in some indicators of different plants are not necessarily the same, whereas a simple comparison of some indicators shows a very scattered or difficultto-interpret pattern, so that we established a correlation between DDI and other indices of leaves and fibrous roots and made use of principal component analysis and subordinate functions to obtain the final result. We also need to mention that although a greenhouse provides more controlled conditions in stress treatments, the relationship between the findings of this greenhouse experiment and a field experiment needs further study and validation.

\section{Literature Cited}

A, R.W., X.G. Sun, L.L. Wang, and T.J. Guo. 2015. Physiological responses of three tree peony cultivars to drought and waterlogging stress. Jiangsu Agr. Sci. 43:252-255.

Aranda, I., F.J. Cano, A. Gascó, H. Cochard, A. Nardini, J.A. Mancha, R. López, and D. Sánchez-Gómez. 2015. Variation in photosynthetic performance and hydraulic architecture across European beech (Fagus sylvatica L.) populations supports the case for local adaptation to water stress. Tree Physiol. 35:34-46.
Barrs, H.D. and P.E. Weatherley. 1962. A reexamination of the relative turgidity technique for estimating water deficits in leaves. Austral. J. Biol. Sci. 15:413-428.

Bates, L.S., R.P. Waldren, and I.D. Teare. 1973. Rapid determination of free proline for waterstress studies. Plant Soil 39:205-207.

Bowler, C., W.V. Camp, M.V. Montagu, D. Inzé, and K. Asada. 1994. Superoxide dismutase in plants. Crit. Rev. Plant Sci. 13:199-218.

Bradford, M.M. 1976. A rapid and sensitive method for the quantitation of microgram quantities of protein utilizing the principle of protein-dye binding. Anal. Biochem. 72:248-254.

Caverzan, A., A. Casassola, and S.P. Patussi Brammer. 2016. Reactive oxygen species and antioxidant enzymes involved in plant tolerance to stress, p. 468-469. In: A. Shanker (ed.). Abiotic and biotic stress in plants - recent advances and future perspectives. InTech, Rijeka, Croatia.

Chen, X.M., L.L. Qiu, H.P. Guo, Y. Wang, H.W Yuan, D.L. Yan, and B.S. Zheng. 2017. Spermidine induces physiological and biochemical changes in southern highbush blueberry under drought stress. Braz. J. Bot. 40:1-11.

Dacosta, M. and B.R. Huang. 2007. Drought survival and recuperative ability of bentgrass species associated with changes in abscisic acid and cytokinin production. J. Amer. Soc. Hort. Sci. 132:60-66.

Davies, P.J. 2010. Plant hormones: Biosynthesis, signal transduction, action! 3rd ed. Springer, Dordrecht, Dutch.

Duncan, D.B. 1955. Multiple range and multiple F-tests. Biometrics 11:1-42.
Egert, M. and M. Tevini. 2002. Influence of drought on some physiological parameters symptomatic for oxidative stress in leaves of chives (Allium schoenoprasum). Environ. Expt. Bot. 48:43-49.

Fan, S.L., Z.H. Yuan, L.J. Feng, X.H. Wang, X.M. Ding, and H.L. Zhen. 2011. Effects of drought stress on physiological and biochemical parameters of Dahlia pinnata. Chin. J. Appl. Ecol. 22:651-657.

Farooq, M., A. Wahid, N. Kobayashi, D. Fujita, and S.M.A. Basra. 2009. Plant drought stress: Effects, mechanisms and management. Agron. Sustain. Dev. 29:185-212.

Guo, H. 2009. Effects of soil drought stress on the physiological and biochemical reaction of four cultivars in Paeonia lactiflora. Academic dissertation, Hunan Agricultural University, China.

Guo, L.L., G.X. Liu, L.N. Wang, J. Li, J.H. Li, X.G. Hou, and X.S. Kong. 2014. Effects of gradual drought and seedling-recovering watering on photosynthetic characteristics of tree peony. Acta Agr. Shanghai 30:105-108.

Heath, R.L. and L. Packer. 1968. Photoperoxidation in isolated chloroplasts: I. Kinetics and stoichiometry of fatty acid peroxidation. Arch. Biochem. Biophys. 125:189-198.

Hossain, Z., M.Z. Nouri, and S. Komatsu. 2012. Plant cell organelle proteomics in response to abiotic stress. J. Proteome Res. 11:37-48.

Kamenetsky, R., A. Barzilay, A. Erez, and A.H. Halevy. 2003. Temperature requirements for floral development of herbaceous peony 'Sarah Bernhardt'. Scientia Hort. 97:309-320.

Kong, X.S., M.X. Zhang, X.Y. Wang, R. Huang, Y.Y. Dong, and S. Lin. 2011. Comparative studies on the physiological and biochemical characteristics of two Paeonia suffruticosa varieties under water stress. Sci. Silvae Sin. 47:162-167.

Lambers, H., F.S. Chapin, and T.L. Pons. 2008. Plant physiological ecology. 2nd ed. Springer, New York, NY.

Li, D.X., C.D. Li, C.F. Sun, H.C. Sun, L.T. Liu, Y.J. Zhang, and K. Xiao. 2010. The effects of drought on endogenous hormone contents and balance in main stem leaves of cotton. Cotton Sci. 22:231-235.

Li, H.S. 2000. The experimental principles and techniques of plant physiology and biochemistry. Higher Education Press, Beijing, China.

Li, J. 2014. The changes of physiological and biochemical characteristic and transcriptome analysis of different varieties of Paeonia suffruticosa under drought stress. Academic dissertation, Henan University of Science and Technology, China.

Li, J.J. 1999. Tree peony and herbaceous peony of China. China's Forestry Press, Beijing, China.

Li, Z., H. Zhou, Y. Peng, X.Q. Zhang, X. Ma, L.K Huang, and Y.H. Yan. 2015. Exogenously applied spermidine improves drought tolerance in creeping bentgrass associated with changes in antioxidant defense, endogenous polyamines and phytohormones. Plant Growth Regulat. 76:71-82.

Mahouachi, J., V. Arbona, and A. Gómez-Cadenas. 2007. Hormonal changes in papaya seedlings subjected to progressive water stress and rewatering. Plant Growth Regulat. 53:43-51.

Morkunas, I., V.C. Mai, A. Waskiewicz, M. Formela, and P. Goliński. 2014. Major phytohormones under abiotic stress, p. 87-135. In: A. Ahmad and M.R. Wani (eds.). Physiological mechanisms and adaptation strategies in plants under changing environment. Springer, New York, NY. 
Morris, D.L. 1948. Quantitative determination of carbohydrates with Dreywood's anthrone reagent. Science 107:254-255.

Peng, M.G., J. Zhang, X.L. Chen, J.P. Zhang, Y.F. Zhang, and Y.X. Bai. 2014. Drought resistance of Paeonia rockii under polyethylene glycol simulated drought stress. J. Northwest A\&F Univ. 42:179-186.

Raviv, M. and T.J. Blom. 2001. The effect of water availability and quality on photosynthesis and productivity of soilless-grown cut roses. Scientia Hort. 88:257-276.

Shao, H.B., L.Y. Chu, G. Wu, J.H. Zhang, Z.H. Lu, and Y.C. Hu. 2007. Changes of some antioxidative physiological indices under soil water deficits among 10 wheat (Triticum aestivum L.) genotypes at tillering stage. Colloids Surf. B Biointerfaces 54:143-149.

Smith, A.M., G. Coupland, L. Dolan, N. Harberd, J. Jones, C. Martin, R. Sablowski, and A. Amey.
2009. Plant biology. Garland Science, New York, NY.

Wang, Q., J.X. Liu, J.J. Zhang, and X.N. Yu. 2014a. The effects of growth and physiology of herbaceous peony under water stress. J. Plant Genet. Resources 15:1270-1277.

Wang, Q., T.R. Cheng, X.N. Yu, J.A. Teixeira da Silva, and D.H. Byrne. 2014b. Physiological and biochemical responses of six herbaceous peony cultivars to cold stress. S. Afr. J. Bot. 94:140-148.

Wang, Q., Y.B. Yuan, and X.N. Yu. 2013. Study on physiological characteristics and saline-alkali tolerance for two cultivars of herbaceous peony. J. Agr. Univ. Hebei 32:52-60.

Yooyongwech, S., T. Samphumphung, R. Tisaram, C. Theerawitaya, and S. Cha-Um. 2017. Physiological, morphological changes and storage root yield of sweetpotato [Ipomoea batatas (L.) Lam.] under PEG-induced water stress.
Not. Bot. Horti Agrobot. Cluj-Napoca 45: 164.

Zhang, C. and S. Shi. 2018. Physiological and proteomic responses of contrasting alfalfa (Medicago sativa L.) varieties to PEGinduced osmotic stress. Front. Plant Sci. 9:242.

Zhang, J.P., D.Q. Li, J.J. Nie, and Y.P. Xia. 2016. Physiological and biochemical responses to the high temperature stress and heat resistance evaluation of Paeonia lactiflora cultivars. J. Nucl. Agr. Sci. 30:1848-1856.

Zhao, D.Q., Z.J. Hao, J. Wang, and J. Tao. 2013. Effects of $\mathrm{pH}$ in irrigation water on plant growth and flower quality in herbaceous peony (Paeonia lactiflora Pall.). Scientia Hort. 154:45-53.

Zhou, F., E.S. Liu, P.J. Zhao, W.Q. Wang, and M. Peng. 2013. Impacts of drought stress on content of endogenous phytohormones at seedling stage of cassava. Agr. Res. Arid Areas $31: 238-244$. 